\title{
On the Equivalence of KMS and Gibbs Conditions for States of Quantum Lattice Systems
}

\author{
Huzihiro Araki and P. D. F. Ion \\ Research Institute for Mathematical Sciences, Kyoto University, Kyoto, Japan 606 \\ Received August 28, 1973

\begin{abstract}
The equivalence of a Gibbsian equilibrium condition and the KMS condition is proven for one-dimensional quantum lattice systems with a finite range interaction at arbitrary temperature, and for quantum lattice systems of arbitrary dimension, with a finite body interaction, at high temperature.
\end{abstract}

\section{$\S 1$. Introduction}

There are a large number of conditions which characterize equilibrium states of infinitely extended lattice systems (see [5] for example). Most of them are known to be mutually equivalent for classical and quantum lattice systems. One exception has been the equivalence of the Gibbsian equilibrium condition and the KMS condition for quantum lattice systems. We shall show this equivalence for one-dimensional quantum lattice systems at all temperatures and for a general class of quantum lattice systems at high temperature.

In Section 2, we shall fix our notation and terminology. In Section 3, we collect some technical estimates. In Section 4, we formulate a Gibbs condition, which becomes equivalent to equilibrium conditions of Lanford and Ruelle, and Dobrushin when the interaction is classical. In Section 5, we derive the equivalence of the Gibbs condition and the KMS condition. In Section 6, we derive the equivalence of the Gibbs condition and the variational principle when the state is invariant under lattice translations.

\section{§ 2. The KMS Condition}

We consider a $v$-dimensional quantum lattice system. For a finite subset $I$ of $\mathbb{Z}^{v}, N(I)$ denotes the number of points in $I$ and $d(I)$ denotes the largest distance between 2 points in $I$.

The $C^{*}$-algebra $\mathfrak{A}$ (of quasi-local observables) contains a sub- $C^{*}$ algebra $\mathfrak{U}(I)$ for each subset $I$ of $\mathbb{Z}$ satisfying the following conditions: 
(1) $\mathfrak{U}(I)$ for each finite subset $I$ is a full matrix algebra of some finite dimension. (2) If $I$ and $I^{\prime}$ are disjoint, $\mathfrak{A}(I)$ and $\mathfrak{A}\left(I^{\prime}\right)$ commute elementwise. (3) If $I$ is the union of $I_{n}$, then $\mathfrak{A}(I)$ is generated (as a $C^{*}$-algebra) by the union of $\mathfrak{U}\left(I_{n}\right)$. For each $Q \in \mathfrak{U}$, we call the infimum (in the set inclusion order) of sets $I$ satisfying $Q \in \mathfrak{A}(I)$ the support of $Q$ and denote it by $\operatorname{supp} Q$. We denote $N(Q) \equiv N(\operatorname{supp} Q), d(Q) \equiv d(\operatorname{supp} Q)$. $\|Q\|_{N \leq m}$ denotes the infimum of $\sum_{i}\left\|Q_{i}\right\|$ such that $Q=\sum_{i} Q_{i}$ and $N(Q) \leqq m$. If such $\left\{Q_{i}\right\}$ does not exist, we define $\|Q\|_{N \leqq m}=\infty$. $\|Q\|_{d \leqq m}$ denotes the infimum of $\sum_{i}\left\|Q_{i}\right\|$ such that $Q=\sum_{i} Q_{i}$ and $d\left(Q_{i}\right) \leqq m$. If such $\left\{Q_{i}\right\}$ does not exist, we define $\|Q\|_{d \leqq m}=\infty$.

A representation $\tau$ of the translation group $\mathbb{Z}^{v}$ by $*$-automorphisms of $\mathfrak{A}$ is supposed to exist such that $\tau(x) \mathfrak{A}(I)=\mathfrak{A}(I+x)$ for every $x \in \mathbb{Z}^{v}$, where $I+x=\{x+a ; a \in I\}$.

For each finite subset $I$ of $\mathbb{Z}$, we are given a self-adjoint element $\Phi(I) \in \mathfrak{A}(I)$, called an interaction potential, satisfying the following conditions: (1) $\Phi$ (empty set) $=0$, (2) $\tau(x) \Phi(I)=\Phi(I+x)$, (3) $\|\Phi\|$ $\equiv \sum_{I \ni 0}\|\Phi(I)\| / N(I)<\infty$. Let $N(\Phi)$ denote the supremum of $N(I)$ such that $\Phi(I) \neq 0$. In the following we shall be interested in $\Phi$ with a finite $N(\Phi)$ (namely the case where $n$-body interaction is absent for large $n$ ). Let $d(\Phi)$ denote the supremum of $d(I)$ such that $\Phi(I) \neq 0$. A finite range interaction is characterized by $d(\Phi)<\infty$.

The energy $U^{\Phi}(I)$ in a finite region $I$ is defined by $U^{\Phi}(I)=\sum_{I^{\prime} \subset I} \Phi\left(I^{\prime}\right)$. We have $\left\|U^{\Phi}(I)\right\| \leqq\|\Phi\| N(I)$. The time translation automorphisms $\sigma_{t}$ for $-\infty<t<\infty$ are defined by

$$
\sigma_{t} Q=\lim _{I \rightarrow \infty} e^{i U^{\Phi}(I) t} Q e^{-i U^{\Phi}(I) t}, \quad Q \in \mathfrak{A}
$$

where the limit is known to exist if $N(\Phi)<\infty$. ([13] or [15]. For a weaker condition on $\Phi$, see [14], p. 193.)

A state $\varphi$ of $\mathfrak{A}$ is defined to be a KMS state with an inverse temperature $\beta$ (in the units where the Boltzmann constant is 1) if $\varphi$ satisfies the following KMS condition: For any elements $Q_{1}$ and $Q_{2}$ in $\mathfrak{A}$, there exists a function $F(z)$ of $z$ in the complex plane which is continuous for $\operatorname{Im} z \in[0, \beta]$, holomorphic for $\operatorname{Im} z \in(0, \beta)$ and satisfies $F(t)=\varphi\left(Q_{2} \sigma_{t} Q_{1}\right), F(t+i \beta)$ $=\varphi\left(\sigma_{t}\left(Q_{1}\right) Q_{2}\right)$.

For each state $\varphi$, there exists a Hilbert space $\mathscr{H}_{\varphi}$, a representation $\pi_{\varphi}$ of $\mathfrak{A}$ on $\mathscr{H}_{\varphi}$ and a cyclic vector $\Omega_{\varphi} \in \mathscr{H}$ such that $\varphi(Q)=\left(\Omega_{\varphi}, \pi_{\varphi}(Q) \Omega_{\varphi}\right)$. (Here an inner product $(x, y)$ is linear in $y$ according to the physicists' convention.) If the state is invariant under time translation (namely $\varphi\left(\sigma_{t}(Q)\right)=\varphi(Q)$ for any $\left.Q \in \mathfrak{U}, t \in \mathbb{R}\right)$, then there exists a strongly continuous unitary representation $U_{\varphi}(t)$ of $t \in \mathbb{R}$ such that $U_{\varphi}(t) \pi_{\varphi}(Q) \Omega_{\varphi}$ $=\pi_{\varphi}\left(\sigma_{t} Q\right) \Omega_{\varphi}$. 
If $\varphi$ is a $\mathrm{KMS}$ state, $\varphi$ is invariant under $\sigma_{t}$. Furthermore the vector $\Omega_{\varphi}$ is cyclic and separating for the von Neumann algebra $\mathfrak{M}=\pi_{\varphi}(\mathfrak{U})^{\prime \prime}$. (The cyclicity by definition, and the separating property by the KMS condition.) It should be added that $\mathfrak{U}$ is simple, $\pi_{\varphi}$ is faithful and hence $\varphi$ is faithful.

By Stone's theorem, there exists a self-adjoint operator $H_{\varphi}$ such that $U_{\varphi}(t)=e^{i H_{\varphi} t}$. The positive self-adjoint operator

$$
\Delta=e^{-\beta H_{\varphi}}\left(=\Delta_{\Omega_{\varphi}}\right)
$$

is called the modular operator for the cyclic and separating vector $\Omega_{\varphi}$ of $\mathfrak{M}$. The automorphism $\sigma_{t}^{\varphi}=\sigma_{-\beta t}$ is called the modular automorphism.

\section{§ 3. Technical Lemmas}

\section{Time Translation}

First we consider the case of arbitrary dimension $v$ and a finite-body interaction $(N(\Phi)<\infty)$. Let $\delta_{A} Q$ denote the commutator $[A, Q]$ and $\delta_{U^{\Phi}(I)} Q \equiv \sum_{I^{\prime} \subset I}\left[\Phi\left(I^{\prime}\right), Q\right]$ for finite or infinite $I$. The sum is norm convergent if $\|Q\|_{N \leqq m}<\infty$ for some $m$.

Lemma 1. If $N(\Phi)<\infty$ and $\|Q\|_{N \leqq m}<\infty$ for some $m$, then

$$
\left\|\left(\delta_{U^{\Phi}(I)}\right)^{n} Q\right\| \leqq\left\{2(N(\Phi)-1)\|\Phi\|^{\prime}\right\}^{n}(n-1) ! a n^{a}\|Q\|_{N \leqq m}
$$

where $\|\Phi\|^{\prime}=\sum_{I \ni 0}\|\Phi(I)\|$ and $a=m /(N(\Phi)-1)$. The power series

$$
\sigma_{t}^{I} Q \equiv \sum_{n=0}^{\infty} n !^{-1}(i t)^{n}\left(\delta_{U^{\Phi}(I)}\right)^{n} Q
$$

is absolutely convergent for

$$
|t|<r(\Phi) \equiv\left\{2(N(\Phi)-1)\|\Phi\|^{\prime}\right\}^{-1}
$$

if $\|Q\|_{N \leqq m}<\infty$ for some $m$. The convergence is uniform for $|t| \leqq T$ and $\|Q\|_{N \leqq m} \leqq M$ if $T<r(\Phi)$. For such $t$ and $Q$,

$$
\left\|\sigma_{t}^{I} Q\right\| \leqq a(T ; m)\|Q\|_{N \leqq m}
$$

for some constant $a(T ; m)$.

The proof is essentially in [13]. We denote $\sigma_{t}^{\mathbb{Z}^{v}}$ by $\sigma_{t}$.

The corresponding Lemma for the case $v=1$ is the following:

Lemma 2. If $v=1, d(\Phi)<\infty$ and $\|Q\|_{d \leqq m}<\infty$, then the power series (3.2) converges absolutely for any $t$ in the complex plane. The convergence is uniform for $|t| \leqq T$ for any $T$ and (3.3) holds for some constant $a(T ; m)$. 
The proof is in [2].

The absolute convergence in the above Lemmas is established for the series (3.2) after the defining summation for $\left(\delta_{U^{\Phi}(I)}\right)^{n} Q$ is substituted. From this it immediately follows that under the conditions of Lemmas 1 and 2

$$
\lim _{I \rightarrow \mathbb{Z}^{v}} \sigma_{t}^{I} Q=\sigma_{t} Q \quad \text { (norm convergence) } .
$$

If $I$ is finite, then

$$
\sigma_{t}^{I} Q=e^{i U^{\Phi}(I) t} Q e^{-i U^{\Phi}(I) t}
$$

If $t$ is real in addition, $\sigma_{t}^{I}$ for finite $I$ obviously extends to a one-parameter group of $*$-automorphisms of $\mathfrak{A}$. Hence $\left\|\sigma_{t}^{I}\right\|=1$ for real $t$. By taking limits, we obtain the same properties for general $I$ and in particular for $\sigma_{t}$; so then (3.4) holds for extended $\sigma_{t}^{I}$ and $\sigma_{t}$.

For real $s$ and $t$, we have $\sigma_{t+s}^{I} Q=\sigma_{t}^{I}\left(\sigma_{s}^{I} Q\right)$ for any $I$ and $Q \in \mathfrak{A}$. By an analytic continuation, the same equation holds for complex $s$ provided that the two sides are definable by (3.2). Hence we may define $\sigma_{t+i s}^{I}$ for real $t$ and $s$ satisfying $|s|<r(\Phi)$ by

$$
\sigma_{t+i s}^{I} Q=\sigma_{t}^{I}\left(\sigma_{i s}^{I} Q\right) \text {. }
$$

\section{Surface Energy}

Let $I^{\prime} \backslash I$ denote the intersection of $I^{\prime}$ and the complement $I^{c}$ of $I$. Suppose $I^{\prime} \supset I$ and let

$$
\begin{gathered}
W_{I, I^{\prime}}=\sum_{\emptyset \neq I_{1} \subset I, \emptyset \neq I_{2} \subset I^{\prime} \backslash I} \Phi\left(I_{1} \cup I_{2}\right), \\
W_{I}=W_{I, \mathbb{Z}^{v}} .
\end{gathered}
$$

$W_{I}$ is the sum of the interaction potential across the boundary of $I$ and its complement.

Lemma 3. As a finite subset $I^{\prime}$ tends to $\mathbb{Z}^{v},\left\|W_{I, I^{\prime}}-W_{I}\right\|$ tends to 0 if $N(I)<\infty$.

Proof. This follows from the absolute convergence of the sum (3.7) for $I^{\prime}=\mathbb{Z}^{v}$ and finite $I$. The latter follows from

$$
\sum\left\|\Phi\left(I_{1} \cup I_{2}\right)\right\| \leqq \sum_{p \in I} \sum_{I^{\prime \prime} \ni p}\left\|\Phi\left(I^{\prime \prime}\right)\right\|=N(I)\|\Phi\|^{\prime} .
$$

Let $h_{a}(I)$ denote the ratio of the maximum number of mutually disjoint translates of a cube of side $a$ which are contained in $I$, and the minimum number of translates of a cube of side $a$ the union of which can cover $I$. When we say that $I$ tends to $\mathbb{Z}^{v}$ in the sense of van Hove, we consider a net of finite subsets $I_{\alpha}$ such that $I_{\alpha}$ tends to $\mathbb{Z}^{v}$ in set inclusion order and $h_{a}\left(I_{\alpha}\right) \rightarrow 1$ for every $a$. 
Lemma 4. If I tends to $\mathbb{Z}^{v}$ in the sense of van Hove, then $\left\|W_{I}\right\|_{N \leqq N(\Phi)} / N(I)$ tends to 0 , provided that $N(\Phi)<\infty$.

Proof. Let $C(a)$ denote the cube of side $2 a$ and center at the origin. $N(C(a))=(2 a+1)^{\nu}$. Since $\|\Phi\|^{\prime}<\infty$, there exists an $a$ for any given $0<\varepsilon<1$ such that $\|\Phi\|^{\prime}-\sum_{C(a) \supset I \ni 0}\|\Phi(I)\|<\varepsilon$. Let the integer $n$ satisfy $1-\left(1-n^{-1}\right)^{v}<\varepsilon$. Let $b=2 a n$. For any finite set $I$, let $I_{\varepsilon}^{\text {in }}$ be the set of points $p$ such that $p+C(a)$ is in $I$. If $h_{b}(I)>1-\varepsilon$, then there exist mutually disjoint translates $J_{k}$ of $C(b)$ contained in $I$ such that

$$
\sum_{k} N\left(J_{k}\right) \geqq h_{b}(I) N(I)>(1-\varepsilon) N(I) .
$$

On the other hand

$$
N\left(\left(J_{k}\right)_{\varepsilon}^{\text {in }}\right)=[\{(n-1) 2 a+1\} /(2 a n+1)]^{v} N\left(J_{k}\right)>(1-\varepsilon) N\left(J_{k}\right) .
$$

Since the point sets $\left(J_{k}\right)_{\varepsilon}^{\text {in }}$ are mutually disjoint and contained in $I_{\varepsilon}^{\text {in }}$, we have

$$
N\left(I_{\varepsilon}^{\mathrm{in}}\right) \geqq \sum_{k} N\left(\left(J_{k}\right)_{\varepsilon}^{\mathrm{in}}\right)>(1-\varepsilon)^{2} N(I) .
$$

Let $I \backslash I_{\varepsilon}^{\text {in }}=I_{\varepsilon}^{\text {sur }}$. Inequality (3.11) implies

$$
N\left(I_{\varepsilon}^{\mathrm{sur}}\right) / N(I)<\varepsilon(2-\varepsilon)
$$

Let

Then

$$
\begin{aligned}
& W_{1}=\sum_{I_{1} \subset I, I_{1} \cap I_{I^{\mathrm{in}} \neq \emptyset}} \sum_{I_{2} \subset I^{c}} \Phi\left(I_{1} \cup I_{2}\right), \\
& W_{2}=\sum_{I_{1} \subset I_{\varepsilon}^{\text {sur }}} \sum_{I_{2} \subset I^{c}} \Phi\left(I_{1} \cup I_{2}\right) .
\end{aligned}
$$

$$
\begin{aligned}
& \left\|W_{1}\right\|_{N \leqq N(\Phi)} \leqq \sum_{p \in I_{\varepsilon}^{\text {in }}} \sum_{I^{\prime} \ni p, I^{\prime} \Varangle p+C(a)}\left\|\Phi\left(I^{\prime}\right)\right\| \leqq N\left(I_{\varepsilon}^{\text {in }}\right) \varepsilon<N(I) \varepsilon, \\
& \left\|W_{2}\right\|_{N \leqq N(\Phi)} \leqq \sum_{p \in I_{\varepsilon}^{\text {sur }}} \sum_{I^{\prime} \ni p}\left\|\Phi\left(I^{\prime}\right)\right\| \leqq N\left(I_{\varepsilon}^{\text {sur }}\right)\|\Phi\|^{\prime} .
\end{aligned}
$$

Since $W_{I}=W_{1}+W_{2}$, we have

$$
\left\|W_{I}\right\|_{N \leqq N(\Phi)} / N(I)<\varepsilon+\varepsilon(2-\varepsilon)\|\Phi\|^{\prime}
$$

which tends to 0 as $\varepsilon \rightarrow 0$.

Lemma 5. If $I$ tends to $\mathbb{Z}^{v}$ in the sense of Van Hove, then $\left\|W_{I}\right\|_{d \leqq d(\Phi)} / N(I)$ tends to 0 , provided that $d(\Phi)<\infty$.

The proof is similar to that of Lemma 4. 


\section{The Intertwiner $\Lambda_{I}^{t}$}

Let

$$
\Lambda_{I, I^{\prime}}^{t}=\operatorname{Exp}_{l}\left(\int_{0}^{1} ; \sigma_{t s}^{I^{\prime}}\left(i t W_{I, I^{\prime}}\right) d s\right)
$$

where the expansional is defined in [3]; (3.13) is well-defined by Lemma 1 if $|\operatorname{Im} t|<r(\Phi)$. By Propositions 3 and 4 of [3], it has an inverse:

$$
\left(\Lambda_{I, I^{\prime}}^{t}\right)^{-1}=\operatorname{Exp}_{r}\left(\int_{0}^{1} ;-\sigma_{t s}^{I^{\prime}}\left(i t W_{I, I^{\prime}}\right) d s\right) .
$$

For $I^{\prime}=\mathbb{Z}^{v}$, we write $\Lambda_{I}^{t}$ instead of $\Lambda_{I, \mathbb{Z}^{\nu}}^{t}$. For finite $I^{\prime}$, we have by Theorem 3 of [3]

$$
\Lambda_{I, I^{\prime}}^{t}=e^{i t u^{\Phi}\left(I^{\prime}\right)} e^{-i t\left(U^{\Phi}\left(I^{\prime}\right)-W_{I, I^{\prime}}\right)} .
$$

Since $U^{\Phi}\left(I^{\prime}\right)-W_{I, I^{\prime}}=U^{\Phi}(I)+U^{\Phi}\left(I^{\prime} \backslash I\right)$, we have for $Q \in \mathfrak{A}(I)$

$$
\Lambda_{I, I^{\prime}}^{t} e^{i t U^{\Phi}(I)} Q=\sigma_{t}^{I^{\prime}}(Q) \Lambda_{I, I^{\prime}}^{t} e^{i t U^{\Phi}(I)} .
$$

When $t$ is real, this equality for an arbitrary $I^{\prime}$ is obtained from the same for finite $I^{\prime}$ by a limit argument. (Note that $\sigma_{t s}^{I^{\prime}}$ for real $t s$ is a $*$-automorphism.) Hence by analytic continuation, (3.16) holds for general $I^{\prime}$ for $Q \in \mathfrak{U}(I)$, finite $I$ and $|\operatorname{Im} t|<r(\Phi)$ if $N(\Phi)<\infty$, and for $Q \in \mathfrak{U}(I)$, finite $I$ and any $t$ if $v=1$ and $d(\Phi)<\infty$. In particular

$$
\begin{gathered}
\Lambda_{I}^{t} e^{i t U^{\Phi}(I)} Q=\sigma_{t}(Q) \Lambda_{I}^{t} e^{i t U^{\Phi}(I)}, \\
e^{-i t U^{\Phi}(I)}\left(\Lambda_{I}^{t}\right)^{-1} \sigma_{t}(Q)=Q e^{-i t U^{\Phi}(I)}\left(\Lambda_{I}^{t}\right)^{-1} .
\end{gathered}
$$

\section{§ 4. Gibbs Condition}

Let $\varphi_{0}^{I}$ denote the central state (i.e. tracial state) of $\mathfrak{A}(I)$. Since $\mathfrak{A}(I)$ is a full matrix algebra of finite dimension if $I$ is finite, we may view $\mathfrak{A}$ as the tensor product $\mathfrak{U}(I) \otimes \mathfrak{U}\left(I^{c}\right)$ [12]. For a state $\varphi$ of $\mathfrak{U}$, we now introduce the following:

Gibbs Condition. For each finite $I \subset \mathbb{Z}^{v}$, there exists a positive linear functional $\varphi^{I^{c}}$ of $A\left(I^{c}\right)$ such that

$$
\varphi(Q)=\left(\varphi_{0}^{I} \otimes \varphi^{I^{c}}\right)\left(e^{-\beta U^{\Phi}(I) / 2} \Lambda_{I}^{*} Q \Lambda_{I} e^{-\beta U^{\Phi}(I) / 2}\right),
$$

where $\Lambda_{I} \equiv \Lambda_{I}^{i \beta / 2}$.

We shall first show that this condition is equivalent to conditions of Lanford and Ruelle [11] and Dobrushin [7,8] when the interaction is classical, namely when each $\Phi(I)$ belongs to an abelian subalgebra $\mathfrak{M}$ of $\mathfrak{A}$ and $\varphi$ is restricted to $\mathfrak{M} ; \mathfrak{M}$ is the algebra generated by number operators at each lattice site. If a state $\varphi$ satisfies (4.1) and the interaction 
is classical then the state is classical too, in the usual sense [6] that the vanishing of the conditional expectation of an operator $Q$ with respect to $\mathfrak{M}, \varphi_{0}(Q \mid \mathfrak{M})=0$, implies $\varphi(Q)=0$. $\left(\hat{Q} \equiv \varphi_{0}(Q \mid \mathfrak{M})\right.$ is defined by the requirements $\hat{Q} \in \mathfrak{M}$ and $\varphi_{0}\left(Q Q_{1}\right)=\varphi_{0}\left(\hat{Q} Q_{1}\right)$ for all $Q_{1} \in \mathfrak{M}$.) This follows from (4.1) for $Q \in \mathfrak{U}(I)$ for any finite $I$ and hence for all $Q \in \mathfrak{A}$. We then restrict $\varphi$ to $\mathfrak{M}$. Since $\sigma_{t}(Q)=Q$ for $Q \in \mathfrak{M}$, we have $\Lambda_{I}=e^{\beta W_{I} / 2}$. So we have as Gibbs condition

$$
\varphi(Q)=\left(\varphi_{0}^{I} \otimes \varphi^{I^{c}}\right)\left(e^{-\beta\left(U^{\Phi}(I)+W_{I}\right)} Q\right), \quad Q \in \mathfrak{M} .
$$

For $Q_{1} \in \mathfrak{A}(I) \cap \mathfrak{M}$, let us consider the conditional expectation

Then

$$
f_{I}\left(Q_{1}\right)=\varphi_{0}^{I}\left(e^{-\beta\left(U^{\Phi}(I)+W_{I}\right)} Q_{1}\right) \in \mathfrak{U}\left(I^{c}\right) \cap \mathfrak{M} .
$$

$$
\varphi\left(Q_{1} \otimes Q_{2}\right)=\varphi^{I^{c}}\left(f_{I}\left(Q_{1}\right) Q_{2}\right)
$$

for $Q_{1} \in \mathfrak{U}(I) \cap \mathfrak{M}, \quad Q_{2} \in \mathfrak{A}\left(I^{c}\right) \cap \mathfrak{M}$. Lanford and Ruelle consider the situation where there is a minimal projection $P_{0}^{I}$ in $\mathfrak{U}(I) \cap \mathfrak{M}$ (the projection onto the no-particle state in $I)$ such that $e^{-\beta\left(U^{\Phi}(I)+W_{I}\right)} P_{0}^{I}=P_{0}^{I}$. Then $f_{I}\left(P_{0}^{I}\right)=\varphi_{0}^{I}\left(P_{0}^{I}\right)$ and (4.4) implies $\varphi^{I^{c}}\left(Q_{2}\right)=\varphi\left(P_{0}^{I}\right)^{-1} \varphi\left(P_{0}^{I} \otimes Q_{2}\right)$. Hence

$$
\varphi\left(Q_{1} \otimes Q_{2}\right)=\varphi\left(P_{0}^{I} \otimes\left\{f_{I}\left(Q_{1}\right) Q_{2}\right\}\right) / \varphi\left(P_{0}^{I}\right) .
$$

The Eq. (4.5) when written in terms of measures on the spectrum of $\mathfrak{M}$, is the equilibrium condition of Lanford and Ruelle.

To be more explicit, for a classical lattice gas the spectrum of $\mathfrak{M}$ may be seen as the space $\mathscr{P}\left(\mathbb{Z}^{v}\right)$ of all subsets of the lattice, and $\varphi$ gives rise to a measure $\mu$ there. The isomorphism of $\mathfrak{M}$ with $L^{\infty}\left(\mathscr{P}\left(\mathbb{Z}^{v}\right), \mu\right)$ may be written, for $Q$ in $\mathfrak{U}(I)$, as

$$
Q=\sum_{X \subset I} Q^{\sim}(X) n(X) \bar{n}(I \backslash X)
$$

where $n(X)=\bigotimes_{x \in X} n(x), n(x)=a^{*}(x) a(x)$ is the number operator at the site $x$, and similarly $\bar{n}(X)=\bigotimes_{x \in X} a(x) a^{*}(x)$. Thus the function $Q^{\sim}$ corresponds with the operator $Q$, so

$$
\begin{aligned}
\varphi(Q) & =\int_{\mathscr{P}\left(\mathbb{Z}^{v}\right)} Q^{\sim}(X) \mu(d X) \\
& =\sum_{X \subset I} \int_{\mathscr{P}\left(I^{c}\right)} Q^{\sim}(X \cup Y) \varrho_{I}(X, d Y),
\end{aligned}
$$

where the latter equality defines $\varrho_{I}(X, d Y)([11]$, Eq. (3.2)). Then we have

$$
\begin{aligned}
\varphi^{I^{c}}\left(Q_{2}\right) & =\varphi\left(P_{0}^{I} \otimes Q_{2}\right) \varphi_{0}^{I}\left(P_{0}^{I}\right)^{-1} \\
& =\varphi_{0}^{I}\left(P_{0}^{I}\right)^{-1} \sum_{X \subset I} \int_{\mathscr{P}\left(I^{c}\right)} P_{0}^{I \sim}(X) Q_{2}^{\sim}(Y) \varrho_{I}(X, d Y) \\
& =\varphi_{0}^{I}\left(P_{0}^{I}\right)^{-1} \int_{\mathscr{P}\left(I^{c}\right)} Q_{2}^{\sim}(Y) \varrho_{I}(\emptyset, d Y) .
\end{aligned}
$$


Now also

$$
f_{I}\left(Q_{1}\right)=\varphi_{0}^{I}\left(P_{0}^{I}\right) \sum_{X \subset I} \exp \left(-\sum_{S \subset X \cup Y: S \cap X \neq \emptyset} \Phi^{\sim}(S)\right) Q_{1}^{\sim}(X \cup Y) .
$$

[The weight coming from $\varphi_{0}^{I}$ in the summation over $X \subset I$ is a constant, which is equal, for example, to the weight for $X=\emptyset$ and the latter is $\left.\varphi_{0}^{I}\left(P_{0}^{I}\right).\right]$ So, if $Q_{1}$ is in $\mathfrak{A}(I)$, Eq. (4.5) becomes

$$
\begin{aligned}
& \sum_{X \subset I} \int_{\mathscr{P}\left(I^{c}\right)} Q_{1}^{\sim}(X) Q_{2}^{\sim}(Y) \varrho_{I}(X, d y) \\
= & \int_{\mathscr{P}\left(I^{c}\right)} f_{I}\left(Q_{1}\right)^{\sim}(Y) Q_{2}^{\sim}(Y) \varrho_{I}(\emptyset, d Y) / \varphi\left(P_{0}^{I}\right) \\
= & \sum_{X \subset I} \int_{\mathscr{P}\left(I^{c}\right)} \exp \left(-\sum_{S \subset X \cup Y: S \cap X \neq \emptyset} \Phi^{\sim}(S)\right) \\
& \cdot Q_{1}^{\sim}(X) Q_{2}^{\sim}(Y) \varrho_{I}(\emptyset, d Y)
\end{aligned}
$$

expressing exactly the condition in (3.10) of [11].

Conversely a classical state $\tilde{\varphi}$ has a unique extension $\varphi$ to the whole algebra $\mathfrak{A}$ under the requirement that for every $Q, \varphi(Q)=\tilde{\varphi}\left(\varphi_{0}(Q \mid \mathfrak{M})\right)$. Then if (4.5) holds we have (4.2) for $Q=Q_{1} \otimes Q_{2}, Q_{1} \in \mathfrak{U}(I) \cap \mathfrak{M}$ and $Q_{2} \in \mathfrak{U}\left(I^{c}\right) \cap \mathfrak{M}$, where $\varphi^{I^{c}}\left(Q_{2}\right)=\varphi\left(P_{0}^{I} \otimes Q_{2}\right)$. Since such $Q$ are total in $\mathfrak{M}$ in the setting of Lanford and Ruelle, (4.2) holds for $Q \in \mathfrak{M}$ and hence (4.1) holds for $Q \in \mathfrak{A}$. This shows the desired equivalence.

The condition given by Dobrushin is known to be equivalent to that given by Ruelle and Lanford.

\section{§ 5. The Equivalence of Gibbs and KMS Conditions}

Theorem 1. When $N(\Phi)<\infty$ and $\beta / 2<r(\Phi)$, or when $v=1, d(\Phi)<\infty$ and $\beta$ is arbitrary, a state $\varphi$ satisfies the Gibbs condition if and only if it satisfies the KMS condition.

Proof. First assume the Gibbs condition (4.1) for $\varphi$. [Our Gibbs condition is formulated only for $\beta / 2<r(\Phi)$ if $v$ is general and $N(\Phi)<\infty$.] Let $Q=Q_{1} \sigma_{i \beta / 2}\left(Q_{2}\right), Q_{1} \in A, Q_{2} \in \mathfrak{U}(I)$. Then by (3.17) with $t=i \beta / 2$,

$$
\sigma_{i \beta / 2}\left(Q_{2}\right) \Lambda_{I} e^{-\beta U^{\Phi}(I) / 2}=\Lambda_{I} e^{-\beta U^{\Phi}(I) / 2} Q_{2} .
$$

By taking adjoints and replacing $Q_{2}{ }^{*}$ by $Q_{2}$, we have

$$
Q_{2} e^{-\beta U^{\Phi}(I) / 2} \Lambda_{I}^{*}=e^{-\beta U^{\Phi}(I) / 2} \Lambda_{I}^{*} \sigma_{-i \beta / 2}\left(Q_{2}\right) .
$$

Using (5.1), the tracial property of $\varphi_{0}^{I}$ and (5.2), we obtain

$$
\varphi\left(Q_{1} \sigma_{i \beta / 2}\left(Q_{2}\right)\right)=\varphi\left(\sigma_{-i \beta / 2}\left(Q_{2}\right) Q_{1}\right)
$$

whenever $Q_{2} \in \mathfrak{A}(I), Q_{1} \in \mathfrak{U}$. By taking the limit, we obtain (5.3) for any $Q_{2}$ with $\left\|Q_{2}\right\|_{N \leqq m}<\infty$. If $Q_{2} \in \mathfrak{U}(I)$, then $\left\|\left(\delta_{U^{\Phi}\left(\mathbb{Z}^{v}\right)}\right)^{n} Q_{2}\right\|_{N \leqq m}<\infty$ for 
$m=n(N(\Phi)-1)+N(I)$. Hence (5.3) holds if $Q_{2}$ is replaced by $\left.(d / d z)^{n} \sigma_{z}\left(Q_{2}\right)\right|_{z=0}$.

For $Q_{2} \in \mathfrak{A}(I), I$ finite, consider the function

$$
f(z)=\varphi\left(\sigma_{z}\left(Q_{2}\right)\right) .
$$

It is well defined, uniformly bounded and holomorphic for $z$ satisfying $|\operatorname{Im} z| \leqq \beta / 2(<r(\Phi))$. By (5.3) with $Q_{1}=1$, we have $f^{(n)}(i \beta / 2)=f^{(n)}(-i \beta / 2)$ for $n=0,1,2, \ldots$. Hence

$$
f(z+i \beta / 2)=f(z-i \beta / 2)
$$

holds for $z$ in some neighbourhood of 0 . By analytic continuation, (5.4) holds in a neighbourhood of real $z$. Hence $f(z)$ can be extended to a periodic entire function. A periodic entire function can be uniformly bounded only when it is a constant. Hence we have $f(z)=f(0)$. Namely $\varphi$ is $\sigma_{t}$-invariant.

We now consider $Q_{1}(f)$, where $Q_{1} \in A, \tilde{f} \in \mathscr{D}(\mathbb{R})$, the space of infinitely differentiable functions of compact support,

$$
f(t)=(2 \pi)^{-1} \int \tilde{f}(p) e^{i p t} d p, \quad \text { and } \quad Q_{1}(f)=\int \sigma_{t}\left(Q_{1}\right) f(t) d t .
$$

We have $\sigma_{t}\left(Q_{1}(f)\right)=Q_{1}\left(f_{t}\right)$ with $f_{t}(s)=f(s-t)$, which holds obviously for real $t$ and has an analytic continuation for arbitrary $t$. By the $\sigma_{t^{-}}$ invariance, we have

$$
\varphi\left(Q_{1}\left(f_{t}\right) \sigma_{s}\left(Q_{2}\right)\right)=\varphi\left(\sigma_{-s}\left(Q\left(f_{t}\right)\right) Q_{2}\right)=\varphi\left(Q_{1}\left(f_{t-s}\right) Q_{2}\right)
$$

which holds for real $s$ and hence by analytic continuation for all $s$ we are considering $[|\operatorname{Im} s|<r(\Phi)$ for general $v$ and $N(\Phi)<\infty$, arbitrary $s$ for $v=1, d(\Phi)<\infty]$.

Substituting $Q_{1}\left(f_{t-i \beta / 2}\right)$ for $Q_{1}$ in (5.3), we obtain

$$
\varphi\left(\sigma_{t-i \beta}\left(Q_{1}(f)\right) Q_{2}\right)=\varphi\left(Q_{2} \sigma_{t} Q_{1}(f)\right) .
$$

By taking limits, this equation holds for arbitrary $Q_{2} \in \mathfrak{A}$. Taking $F(z)$ to be $\varphi\left(Q_{2} \sigma_{z}\left[Q_{1}(f)\right]\right)$, we have $F(t)=\left(Q_{2} \sigma_{t}\left(Q_{1}\right)\right)$ and $F(t+i \beta)$ $=\left(\sigma_{t}\left[Q_{1}(f)\right] Q_{2}\right)$, which is the KMS condition for operators $Q_{1}(f)$ and $Q_{2}$.

It is known that this implies the KMS condition for any $Q_{1}$ and $Q_{2}$ in $\mathfrak{U}$ (see Lemma 2.2 of [1], for example).

We now prove the converse. Assume the KMS condition for $\varphi$. We consider arbitrary $\beta$ when $v=1$ and $d(\Phi)<\infty$, and $\beta$ satisfying $\beta / 2<r(\Phi)$ when $v$ is general and $N(\Phi)<\infty$. Let

$$
\hat{\varphi}_{I}(Q)=\varphi\left(\left(\Lambda_{I}^{*}\right)^{-1} e^{\beta U^{\Phi}(I) / 2} Q e^{\beta U^{\Phi}(I) / 2} \Lambda_{I}^{-1}\right) .
$$

By the KMS condition one has

$$
\varphi\left(B \sigma_{i \beta / 2}\left(Q_{1}\right)\right)=\varphi\left(\sigma_{-i \beta / 2}\left(Q_{1}\right) B\right)
$$


for $Q_{1} \in \mathfrak{A}(I)$ and $B \in \mathfrak{U}$, where $I$ is finite. By (3.18), we have

$$
Q_{1} e^{\beta U^{\Phi}(I) / 2} \Lambda_{I}^{-1}=e^{\beta U^{\Phi}(I) / 2} \Lambda_{I}^{-1} \sigma_{i \beta / 2}\left(Q_{1}\right) \text {. }
$$

By taking the adjoint and replacing $Q_{1} *$ by $Q_{1}$, we also have

$$
\sigma_{-i \beta / 2}\left(Q_{1}\right)\left(\Lambda_{I}^{*}\right)^{-1} e^{\beta U^{\Phi}(I) / 2}=\left(\Lambda_{I}^{*}\right)^{-1} e^{\beta U^{\Phi}(I) / 2} Q_{1} \text {. }
$$

Hence we have

$$
\hat{\varphi}_{I}\left(Q_{2} Q_{1}\right)=\hat{\varphi}_{I}\left(Q_{1} Q_{2}\right)
$$

for any $Q_{2} \in \mathfrak{U}, Q_{1} \in \mathfrak{U}(I)$, which implies $\hat{\varphi}_{I}=\varphi_{0}^{I} \otimes \varphi^{I^{c}}$ where $\varphi^{I^{c}}$ is the restriction of $\hat{\varphi}_{I}$ to $\mathfrak{A}\left(I^{c}\right)$. Solving (5.6) for $\varphi$, we obtain the Gibbs condition.

Q.E.D.

\section{$\S$ 6. Equivalence of the Gibbs Condition with the Variational Principle for Translationally Invariant States}

Theorem 2. A state $\varphi$, invariant under the lattice translations $\tau(a)$, satisfies the Gibbs condition if and only if it satisfies the variational principle:

$$
s(\varphi)-\beta \varphi\left(A_{\Phi}\right) \geqq s(\psi)-\beta \psi\left(A_{\Phi}\right)
$$

for all translationally invariant states $\psi$, where

$$
A_{\Phi}=\sum_{I \ni 0} \Phi(I) / N(I)
$$

and $s(\varphi)$ is the mean entropy of $\varphi([9])$. $\beta$ is arbitrary when $v=1, d(\Phi)<\infty$ and $\beta$ is restricted to be in $[0,2 r(\Phi))$ when $v$ is arbitrary and $N(\Phi)<\infty$.

Proof. It is known that the limit

$$
P(\Phi)=\lim N(I)^{-1} \log \varphi_{0}^{I}\left(e^{-\beta U^{\Phi}(I)}\right)
$$

as $I$ tends to $\mathbb{Z}^{v}$ in the sense of van Hove satisfies

$$
P(\Phi)=\sup \left(s(\psi)-\beta \psi\left(A_{\Phi}\right)\right)
$$

where the supremum is over all translationally invariant states $\psi$.

We now assume the KMS condition for a state $\varphi$ and show that $s(\varphi)-\beta \varphi\left(A_{\Phi}\right)=P(\Phi)$. Let

$$
\varphi_{I}(Q) \equiv \varphi\left(\left(\Lambda_{I}^{*}\right)^{-1} Q \Lambda_{I}^{-1}\right) .
$$

By the Gibbs condition, we have

$$
\varphi_{I}(Q)=c \varphi_{0}^{I}\left(e^{-\beta U^{\Phi}(I)} Q\right)
$$

for any $Q \in \mathfrak{U}(I)$, where the constant $c$ can be determined as

$$
c=\varphi_{0}^{I}\left(e^{-\beta U^{\Phi}(I)}\right)^{-1} \varphi_{I}(1) \text {. }
$$

Let $\varrho_{I}(\psi) \in \mathfrak{U}(I)^{+}$be defined by

$$
\psi(Q)=\varphi_{0}^{I}\left(\varrho_{I}(\psi) Q\right), \quad Q \in \mathfrak{U}(I) .
$$


The mean entropy is defined by

$$
s(\psi)=-\lim N(I)^{-1} \psi\left(\log \varrho_{I}(\psi)\right) .
$$

[This definition differs by a constant from the definition using the matrix trace instead of the normalized trace $\varphi_{0}^{I}$. However our definition (6.3) of $P(\Phi)$ is also in terms of $\varphi_{0}^{I}$ and hence (6.4) holds.] From (6.5) we have

$$
\varrho_{I}\left(\varphi_{I}\right)=\varphi_{0}^{I}\left(e^{-\beta U^{\Phi}(I)}\right)^{-1} e^{-\beta U^{\Phi}(I)} \varphi_{I}(1) .
$$

Let $\mathscr{H}_{\varphi}, \pi_{\varphi}$ and $\Omega_{\varphi}$ be associated with $\varphi$ by the GNS construction. Then $\varphi_{I}$ is the vector state whose vector is

$$
\Phi \equiv \pi_{\varphi}\left(\Lambda_{I}^{-1}\right) \Omega_{\varphi}=\operatorname{Exp}_{r}\left(\int_{0}^{1 / 2} ; \sigma_{-i s}^{\varphi}\left(\beta W_{I}\right) d s\right) \Omega_{\varphi}=\Omega_{\varphi}\left(\beta W_{I}\right)
$$

where $\Omega_{\varphi}(h)$ for a relative Hamiltonian $h$ is defined in [5]. By Proposition 4.1 of [5], $\Omega_{\varphi}\left(\beta W_{I}\right) \in V_{\Omega_{\varphi}}$. Hence by Theorem 12 of [4] we have

$$
\varphi_{I} \leqq\left\|\Lambda_{I}{ }^{-1}\right\|^{2} \varphi, \quad \varphi \leqq\left\|\Lambda_{I}\right\|^{2} \varphi_{I} .
$$

[The latter follows from $\Omega_{\varphi}=\pi_{\varphi}\left(\Lambda_{I}\right) \Phi$ and $V_{\Phi}=V_{\Omega_{\varphi}}$ ]

The above inequalities when restricted to $\mathfrak{U}(I)$ yield

$$
\left\|\Lambda_{I}^{-1}\right\|^{-2} \varrho_{I}\left(\varphi_{I}\right) \leqq \varrho_{I}(\varphi) \leqq\left\|\Lambda_{I}\right\|^{2} \varrho_{I}\left(\varphi_{I}\right)
$$

Since $\log \varrho$ is operator monotone for positive $\varrho$,

Since

$$
-2 \log \left\|\Lambda_{I}{ }^{-1}\right\| \leqq \log \varrho_{I}(\varphi)-\log \varrho_{I}\left(\varphi_{I}\right) \leqq 2 \log \left\|\Lambda_{I}\right\| .
$$

and

$$
\begin{gathered}
\log \varrho_{I}\left(\varphi_{I}\right)=-\beta U^{\Phi}(I)-\log \varphi_{0}^{I}\left(e^{-\beta U^{\Phi}(I)}\right)+\log \varphi_{I}(1), \\
\left\|\Lambda_{I}\right\|^{-2} \leqq \varphi_{I}(1) \leqq\left\|\Lambda_{I}^{-1}\right\|^{2} \quad(\text { due to } \varphi(1)=1),
\end{gathered}
$$

$$
\lim N(I)^{-1} \varphi\left(U^{\Phi}(I)\right)=\varphi\left(A_{\Phi}\right)
$$

for translationally invariant $\varphi$, we obtain

$$
s(\varphi)-\beta \varphi\left(A_{\Phi}\right)-P(\Phi)=0
$$

if we prove

$$
\begin{aligned}
& \lim N(I)^{-1} \log \left\|\Lambda_{I}^{-1}\right\|=0, \\
& \lim N(I)^{-1} \log \left\|\Lambda_{I}\right\|=0 .
\end{aligned}
$$

By Lemma 1 for $N(\Phi)<\infty,|s| \leqq T<r(\Phi)$, and by Lemma 2 for $v=1, d(\Phi)<\infty,|s| \leqq T$, we have

$$
\left\|\sigma_{i s}\left(W_{I}\right)\right\| \leqq a(T, k(\Phi))\left\|W_{I}\right\|_{k \leqq k(\Phi)},
$$


where $k=N$ for general $v$, and $k=d$ for $v=1$. Hence

$$
\begin{aligned}
\log \left\|\Lambda_{I}\right\| & \leqq(\beta / 2) a(\beta / 2, k(\Phi))\left\|W_{I}\right\|_{k \leqq k(\Phi)}, \\
\log \left\|\Lambda_{I}{ }^{-1}\right\| & \leqq(\beta / 2) a(\beta / 2, k(\Phi))\left\|W_{I}\right\|_{k<k(\Phi)} .
\end{aligned}
$$

Since $\left\|\Lambda_{I}^{-1}\right\|^{-1} \leqq\left\|\Lambda_{I}\right\|$, we have

$$
\begin{array}{r}
\left|\log \left\|\Lambda_{I}\right\|\right| \leqq(\beta / 2) a(\beta / 2, k(\Phi))\left\|W_{I}\right\|_{k \leqq k(\Phi)}, \\
\left|\log \left\|\Lambda_{I}^{-1}\right\|\right| \leqq(\beta / 2) a(\beta / 2, k(\Phi))\left\|W_{I}\right\|_{k \leqq k(\Phi)} .
\end{array}
$$

By Lemmas 4 and 5, we have (6.6) and (6.7).

It is known that a state satisfying the variational principle satisfies the KMS condition [10].

Q.E.D.

Acknowledgement. The second author (P.D.F.I.) is most grateful to the Research Institute for Mathematical Sciences for the hospitality shown him, and to the Royal Society for the fellowship under the Royal Society Japan Programme which made his stay there possible.

\section{References}

1. Araki, H.: Publ. RIMS 4A, 361-371 (1968)

2. Araki, H.: Commun. math. Phys. 14, 120-157 (1969)

3. Araki, H.: Ann. Sci. École Norm. Sup. 6, 67-84 (1973)

4. Araki, H.: Preprint RIMS 120. To appear in Pacific J. Math.

5. Araki, H.: Publ. RIMS 9, 165-209 (1973)

6. Brascamp, H. J.: Commun. math. Phys. 18, 82-96 (1970)

7. Dobrushin, R. L.: Theor. Prob. Appl. 12 (4), 535-559 (1967); - Teor. Veroyatnost Primen. 12, 595-618 (1967)

8. Dobrushin, R.L.: Funkcional. Anal. i Priložen. 2 (4), 31- 43 (1968); - Functional Anal. Appl. 2, 292-301 (1968)

9. Lanford III, O.E., Robinson, D. W.: J. Math. Phys. 9, 1120-1125 (1968)

10. Lanford III, O.E., Robinson, D.W.: Commun. math. Phys. 9, 327-338 (1968)

11. Lanford III, O. E., Ruelle, D.: Commun. math. Phys. 13, 194-215 (1969)

12. Powers, R. T.: Ann. Math. 86 (1), 138-171 (1967)

13. Robinson, D. W.: Commun. math. Phys. 7, 337-348 (1968)

14. Ruelle, D.: Statistical Mechanics, New York-Amsterdam: Benjamin 1969

15. Streater, R.F.: Commun. math. Phys. 6, 233-247 (1967)

Huzihiro Araki

P. D. F. Ion

Research Institute

for Mathematical Sciences

Kyoto University

Kyoto, Japan 606 